\title{
Análise da ansiedade pré-competitiva e competitiva de jovens judocas
}

\author{
Giovanna Carla INTERDONATO ${ }^{1}$, Bianca MIARKA²* \& Emerson FRANCHINI² \\ ${ }^{1}$ Center of Physical Education and Sport. State University of Londrina (Brazil). \\ ${ }^{2}$ Combat Sports and Martial Arts Research Group. Physical Education and Sport School. University of \\ São Paulo (Brazil).
}

Recepción: 16/10/2013; Aceptación: 14/12/2013; Publicación: 19/12/2013.

\section{Resumo}

ARTÍCULO ORIGINAL

Objetivo: Este estudo objetivou verificar os níveis de ansiedade-traço em judocas, da classe juvenil (entre 14 e 18 anos) no período pré-competitivo e competitivo.

Método: Para tanto, foram analisados 40 atletas (20 de cada sexo) com $5 \pm 2$ anos de prática de Judô. 0 instrumento utilizado foi o "Sport Competition Anxiety Test" (SCAT). Para observar diferenças, utilizou-se Friedman e Mann-Whitney, $\mathrm{p} \leq$ 0,05. Para verificar correlações, usou-se o coeficiente de correlação de Spearman.

Resultados: Os resultados demonstraram que homens e mulheres em competição apresentaram maior ansiedade em relação ao período de treinamento. Quando comparados os gêneros em interação com o momento, o grupo feminino competitivo demonstrou maior ansiedade que os demais grupos. Foram constatadas correlações negativas e significantes entre a ansiedade, nos momentos pré-competitivo e competitivo, com o tempo de prática da modalidade.

Conclusões: Esses dados sugerem que o trabalho preparatório durante o dia da competição deva ser diferente entre o masculino e o feminino, pois enquanto no feminino seria preciso dar atenção ao controle da ansiedade, no masculino outros aspectos poderiam ser analisados/considerados na preparação do atleta no dia da competição.

Palavras-chave: Ansiedade; judô; competição; adolescentes.

\section{Analysis of pre-competitive and competitive anxiety in youth judoka}

\section{Abstract}

Aim: The present study aimed to verify levels of trait anxiety in juvenile judo athletes (between 14 and 18 yrs.) in pre-competitive and competitive moments.

Method: Therefore, 40 athletes (20 boys and 20 girls) with $5 \pm 2$ yrs. of judo practice were analyzed. The instrument used was the "Sport Competition Anxiety Test" (SCAT). In statistical analysis, we observed differences with Friedman and Mann-Whitney tests, $\mathrm{p} \leq$ 0.05 . The Spearman correlation coefficient was used to identify the relationship between variables.

Results: Results demonstrated that males and females presented higher anxiety in championship, when compared with the training period. When compared gender in interaction with the moment, the competitive female group demonstrated higher anxiety than other groups. A significant and negatives correlations between anxiety, during championship and precompetitive moments, and judo practice time were found.

Conclusions: These data suggest preparatory work during the championship day should be different for male and female athletes, while female athletes have to
Análisis de la ansiedad precompetitiva y competitiva en jóvenes judokas

\section{Resumen}

Objetivo: El objetivo del presente estudio fue verificar los niveles de ansiedad-rasgo en judokas de categoría juvenil (entre 14 y 18 años) durante el periodo pre-competitivo y en competición.

Método: Se analizó a 40 atletas (20 de cada género) con $5 \pm$ 2 años de experiencia em judo. El instrumento utilizado fue el "Sport Competition Anxiety Test" (SCAT). Para observar las diferencias se utilizaron los test de Friedman y MannWhitney, $\mathrm{p} \leq 0.05$. Para verificar las correlaciones se utilizó el coeficiente de correlación de Spearman.

Resultados: Los resultados mostraron que hombres y mujeres presentaron mayor ansiedad en competición que en periodos de entrenamiento. La interacción entre género y momento mostró que el grupo femenino en competición tenía más ansiedad que el resto de grupos. Se constataron correlaciones negativas y significativas entre la ansiedad, en los momentos pre-competitivos y competitivos, y el tiempo de práctica de los sujetos en la modalidad.

Conclusiones: Estos datos sugieren que el trabajo preparatorio durante el día de la competición debería ser diferente entre hombres y mujeres. En las mujeres sería preciso atender al control de la ansiedad, mientras que en

\footnotetext{
* E-mail: miarkasport@hotmail.com. Dirección: Universidade de São Paulo - USP. Escola de Educação Física e Esporte. Departamento de Esporte. Av. Prof. Mello Moraes, 65. Cidade Universitária. 05508900. Sao Paulo, Brasil. Http://esportesdecombate.blogspot.com.
} 
control anxiety, in the male group other aspects could be analyzed/considered in the preparation of the athlete.

Keywords: Anxiety; judo; competition; adolescents. el caso de los hombres podrían analizarse/considerarse otros aspectos diferentes para el día de la competición.

Palabras clave: Ansiedad; judo; competición; adolescentes.

\section{1.- Introducción}

A excelência nos confrontos de judô está vinculada a um conjunto de fatores físicos (Franchini et al., 2005, 2011), técnico-táticos (Franchini et al., 2008; Miarka et al., 2010, 2012) e com o desenvolvimento de habilidades psicológicas, perceptivas e cognitivas contextualizadas (Lech, Sterkowicz, \& Rukasz, 2007). Por ser um esporte situacional, componentes estratégicos da luta exigem estados psicológicos ótimos a fim de garantir tomadas de decisões eficazes (Filaire et al., 2001).

Todavia, diferentes variáveis influenciam o estado de prontidão ótimo, dependendo da fase de treinamento do lutador. Por sua vez a apreensão e preocupação podem afetar negativamente o estado ótimo de prontidão e prejudicar o desempenho competitivo. Tais respostas podem ser causadas momentaneamente pela percepção dos estímulos ambientais com expectativa de perigo, ameaça ou desafio existente, rotuladas pela literatura como ansiedade (Figueiredo, 2000; De Rose, 2002; Detanico \& Santos, 2005; Bertuol \& Valentini, 2006; Interdonato et al., 2010; Ferreira, Leite, \& Nascimento, 2010).

Para descrever e compreender melhor o fenômeno da ansiedade, Cattell e Scheier (1961) classificaram a ansiedade em dois tipos, a ansiedade-estado que é o fato de estar ansioso e a ansiedade-traço que representa o fato de ser ansioso. Mais precisamente, segundo Spielberger (1972), a ansiedade-estado é uma condição transitória de tensão percebida pelo indivíduo em resposta a determinados eventos; logo, a ansiedade-traço remete a diferenças individuais relativamente estáveis, ou seja, uma predisposição do sujeito em ser ansioso.

Investigações sobre psicologia do esporte têm dado grande enfoque à ansiedade em competições de modalidades esportivas de combate (Franchini \& Rubio, 2001; Detanico \& Santos, 2005). Isso ocorre em razão de modalidades esportivas que envolvem contato físico, como é o caso das modalidades de combate, possuírem a presença desse estado emocional aparentemente comum em atletas (Franchini \& Rubio, 2001; De Rose et al., 2004). Chapman, Lane, Brieley e Terry (1997), ao analisarem os níveis de ansiedade de 142 competidores de taekwondo por meio do questionário Sport Competition Anxiety Test (SCAT) observaram que os campeões relataram menor ansiedade e maior auto-confiança do que atletas não vencedores. Essa análise revelou que $62,68 \%$ dos participantes poderiam ser classificados previamente como vencedores ou perdedores, com base nos resultados do SCAT. Morales et al. (2013) observaram que atletas de judô de nível internacional apresentaram menor ansiedade somática e menor ansiedade cognitiva, mensuradas via Inventário de Ansiedade-estado Competitiva Revisado (Revised Competitive State Anxiety Inventory, CSAI-2R), em relação a atletas de nível nacional espanhol.

Apesar de ser um assunto de importância comum a técnicos e pesquisadores, ainda existem poucas investigações que revelaram diferenças entre os gêneros em situações pré-competitivas e competitivas. Detanico e Santos (2005) relataram que atletas brasileiros de judô do sexo feminino apresentavam maiores índices de ansiedade quando comparadas com atletas do sexo masculino. Essa investigação ainda revelou que mesmo na classe sênior, na qual os atletas de judô possuem faixa etária maior, aqueles com maior idade possuíam menor ansiedade-traço pré-competitiva quando comparados aos demais. Entretanto, não se conhece as manifestações dos níveis de ansiedade em outras classes com menor idade em período competitivo ou pré-competitivo.

0 jovem inserido no esporte deverá competir quando atingir seu estado de prontidão competitiva, que acontece a partir do equilíbrio de fatores como o crescimento, o desenvolvimento e a maturação sexual. Definir uma idade torna-se difícil devido às diferenças individuais. Estudos que têm como tema principal a ansiedade esportiva comparada à faixa etária têm mostrado que os 
grupos mais jovens apresentam níveis de ansiedade maiores pelo fato de serem mais inexperientes (Interdonato, 2010).

Este conhecimento é de grande importância, dado que recentemente foi estabelecido os Jogos Olímpicos da Juventude, no qual o judô é modalidade oficial (Judge, Petersen, \& Lydum, 2009), bem como houve o restabelecimento do Campeonato Mundial Juvenil da modalidade (IJF, 2010). Assim, é bastante provável que os treinamentos e as competições, nesta faixa etária e anteriores, sejam intensificados, embora exista crítica a esse processo (Julio et al., 2011). Atrelado a essas questões, esse trabalho teve como objetivo analisar os níveis de ansiedade durante o período pré-competitivo e o período competitivo em atletas da classe juvenil de judô de cada um dos sexos.

\section{2.- Materiais e métodos}

O estudo foi do tipo transversal e caracterizou-se como descritivo-observacional, exploratório. Seguindo a resolução 196/96 do Conselho Nacional de Saúde, o respectivo projeto foi encaminhado e aprovado pelo Comitê de Ética em Pesquisa com seres humanos da Universidade Estadual de Londrina - PR.

\section{1.- Amostra}

A amostra foi composta por atletas de judô que competiram no Campeonato Estadual Paranaense de 2010 na cidade de Foz do Iguaçu - PR, disputando nas categorias individuais, em suas respectivas categorias. Foram voluntários 20 atletas do sexo feminino e 20 do sexo masculino com idade (média e desvio padrão) de 15,25 $\pm 0,73$ anos e com tempo de $5 \pm 2$ anos de prática de Judô, este atletas disputam competições de níveis estaduais e nacionais. Todos os participantes e seus responsáveis assinaram o termo de consentimento livre e esclarecido.

\section{2.- Procedimentos}

Os níveis de ansiedade competitiva foram estabelecidos por meio do Sport Competition Anxiety Test (SCAT), desenvolvido por Martens (1977) e validado com um $\alpha$ de Cronbach de 0,77 de fiabilidade por Rollo (2003). Esse questionário foi aplicado dois meses antes da competição e no dia da competição. Este teste avalia os níveis de ansiedade-traço competitiva, e é composto por 15 perguntas que descrevem como o indivíduo se sente em dada situação. A classificação é apresentada em escores, sendo oito itens de ativação, dois de desativação e cinco de efeito placebo. A pontuação é de um a três para os itens de ativação, e de três a um para os de desativação, sendo que o escore e a classificação seguiram o protocolo de Martens (1977) e a classificação: i) 10 até 12 pontos, ansiedade baixa; ii) 13 até 16 pontos, abaixo da média; iii) 17 até 23 pontos, média; iv) 24 até 27 pontos, acima da média, e; v) > 28 pontos, ansiedade alta.

A aplicação do Instrumento de Ansiedade-traço foi realizada para cada atleta pertencente à amostra, tendo sido preenchido voluntariamente, seguindo o protocolo utilizado por De Rose Junior (1985). Os atletas responderam aos questionários individualmente, em duas etapas. A primeira foi realizada dois meses antes do período de competições e a outra no dia da Competição Estadual, 15 minutos antes de lutarem.

\subsection{Análise estatística}

No plano estatístico, foi utilizado o pacote Statistical Package for Science Social (SPSS) versão 20.0 for Windows para realizar análises descritivas, com médias e desvio padrão dos níveis de ansiedade observados. Para os dados não-paramétricos, os testes de Friedman e Mann-Whitney foram conduzidos a fim de comparar os grupos separados em sexo e momento. Posteriormente, calculou-se a medida do tamanho do efeito para a análise não-paramétrica, definido como $\mathrm{R}=\mathrm{Z} / \sqrt{ }$ $\mathrm{N}$, em que r representa o tamanho do efeito, $\mathrm{Z}$ é derivado a partir da conversão de Mann-Whitney e $\mathrm{N}$ é o número total de observações. Esta análise considera valores $\mathrm{r}$ como: o tamanho pequeno 
efeito $(r=0,10)$, o efeito de dimensão média $(r=0,30)$ ou o grande tamanho do efeito $(r=0,50)$. Para identificar o relacionamento entre as variáveis usou-se o coeficiente de correlação de Spearman. Em todos os casos foram adotados níveis de significância $\mathrm{p} \leq 0,05$.

\section{3.- Resultados}

A Tabela 1 apresenta os valores descritivos e as diferenças da ansiedade-traço nos dois momentos da temporada dos atletas de judô masculino e feminino da classe juvenil.

Tabela 1. Níveis de ansiedade pré-competitiva e competitiva em atletas masculinos e femininos de judô pré-competição e na competição.

\begin{tabular}{lccc}
\hline \multicolumn{1}{c}{ Grupos } & Mínimo & Máximo & Média \pm DP \\
\hline Mulheres em momento pré-competitivo $^{\text {a }}$ & 15,0 & 25,0 & $20,7 \pm 2,7$ \\
Mulheres em momento competitivo $^{\text {Homens em momento pré-competitivo }}{ }^{\text {a }}$ & 13,0 & 29,0 & $25,1 \pm 3,3$ \\
Homens em momento competitivo* $^{*}$ & 17,0 & 25,0 & $21,0 \pm 2,1$ \\
\hline
\end{tabular}

Os grupos com a são diferentes do grupo mulheres em momento competitivo, com $\mathrm{p} \leq 0,0001 ; \mathrm{o}$ grupo com* é diferente do grupo mulheres em momento competitivo com $\mathrm{p}=0,025$.

Foram identificados efeitos de gênero $(K=4,769, p<0,029 ; r=-0,29)$ e momento $(K=$ $19,972, \mathrm{p}<0,001 ; \mathrm{r}=-0,68)$ interação entre o momento de mensuração e o gênero $(\mathrm{Q}=12,5, \mathrm{p}<$ 0,$001 ; r=10,27$ ) na ansiedade. 0 teste de Mann Whitney indicou que as atletas do sexo feminino apresentaram valores mais elevados de ansiedade na competição, com $25 \pm 3$ pontos, classificadas como ansiedade acima da média, valor maior que o observado em relação ao período de treinamento, classificado como ansiedade média $(\mathrm{p}<0,001)$. 0 grupo feminino, na condição competitiva, apresentou valores mais elevados de ansiedade em relação ao grupo masculino tanto na condição pré-competitiva, com ansiedade classificada como média $(\mathrm{p}<0,001)$ quanto na condição competitiva, com classificação média $(\mathrm{p}<0,001)$.

A Tabela 2 apresenta os resultados da correlação de Spearman entre as variáveis momento pré-competitivo, competitivo e tempo de judô.

Tabela 2. Dados descritivos da amostra com correlações de Spearman entre as variáveis momento précompetitivo, competitivo e tempo de judô.

\begin{tabular}{lcccccc}
\hline \multicolumn{1}{c}{ Variável } & Mínimo & Máximo & Média \pm DP & Pré-competitivo & Competitivo & Tempo de prática \\
\hline Pré-competitivo & 15,0 & 25,0 & $20,9 \pm 2,4$ & $1^{\mathrm{a}}$ & 0,267 & $-0,064$ \\
Competitivo & 13,0 & 29,0 & $23,8 \pm 3,0$ & 0,267 & $1^{\mathrm{a}}$ & $-0,534^{*}$ \\
Tempo de prática & $1,0^{\mathrm{a}}$ & 3,0 & $1,9 \pm 0,7$ & $-0,064$ & $-0,534^{*}$ & $1^{\mathrm{a}}$ \\
\hline
\end{tabular}

Os valores a diferentes de 0 com $\mathrm{p} \leq 0,0001$; os valores com ${ }^{*}$ são diferentes de 0 com p=0,020.

Quando o grupo como um todo foi considerado, foram constatadas correlações negativas e significantes entre a ansiedade, nos momentos pré-competitivo e competitivo, com o tempo de prática da modalidade.

\section{4.- Discusión}

O principal resultado desta investigação foi que as atletas do sexo feminino apresentaram valores mais elevados de ansiedade na competição em relação ao período de treinamento e mais elevados no período competitivo em relação ao grupo masculino tanto na condição pré-competitiva quanto na condição competitiva. Para medir ansiedade em momento de treinamento e competitivo e, ao mesmo tempo, tentar isolar ao máximo as variáveis que explicariam possíveis modificações nos valores de ansiedade, optou-se em utilizar o Sport Competition Anxiety Test, instrumento prévio à modificação realizada por Martens (1990) que é direcionada especialmente para o momento 
competitivo e engloba outras situações que explicam outro fator que não é ansiedade. Quando observados os resultados do presente estudo, os mesmos indicam que apenas na condição précompetitiva, o grupo feminino apresentou valores inferiores ao grupo masculino na condição competitiva. Não houve diferença entre os grupos no período pré-competitivo. Adicionalmente, foram constatadas correlações significantes, porém fracas, entre a ansiedade na condição de treinamento e na competição. Apesar de plausível, a modificação da ansiedade de acordo com a exposição ao contexto de competição tem sido pouco estudada. Por isso, o presente resultado traz importantes implicações ao conhecimento sobre a modificação do sintoma de ansiedade em relação à carga de estresse competitivo em homens e mulheres.

Moraes (1990) indica que altos níveis de ansiedade podem distorcer a percepção externa do atleta, alterando a capacidade de tomada de decisão durante a competição. No presente estudo, os dados obtidos demonstraram que lutadores jovens de judô possuem índices de ansiedade acima da média, especialmente no período competitivo. Quando comparadas com outros estudos, as médias obtidas com atletas de judô em período pré-competitivo foram mais elevadas que as encontradas em atletas de outras modalidades (Interdonato et al., 2010; De Rose, 2002; Martens, Vealey, \& Burton, 1990), principalmente em modalidades coletivas (De Rose, 2002). Corroborando com os resultados do grupo feminino em nosso estudo, investigação de Santos (2004) demonstrou que alguns lutadores possuem menor ansiedade em treinamentos do que em competições. De acordo com Mahn (2009) os níveis de ansiedade pouco acima do ótimo são desfavoráveis para atividades que requerem habilidades complexas e de precisão, como o judô. Não há uma regra geral sobre o caráter facilitador ou inibidor da ansiedade. Esta parece ter uma influência ora positiva, ora negativa no rendimento, onde outras variantes podem atuar, tais como: a idade do atleta, sua modalidade esportiva e o caráter coletivo ou individual do esporte, dentre outros.

Nossos achados de maior ansiedade no grupo feminino em relação ao grupo masculino corroboram com aqueles de De Rose (2002), que reportou maior ansiedade em tenistas do sexo feminino em comparação com tenistas do sexo masculino. Martens, Vealey e Burton (1990) também revelaram diferença entre os sexos nos índices de ansiedade em diferentes modalidades esportivas. Detanico e Santos (2005) corroboraram esses indicativos no judô, ao analisarem a ansiedade-traço no momento pré-competitivo de lutadores, dado que também verificaram que as judocas mulheres eram significativamente mais ansiosas que os homens. Na mesma perspectiva o estudo realizado por Gonçalves e Belo (2007) encontrou que a ansiedade traço em atletas do sexo feminino de diferentes modalidades esportivas foram maiores do que em atletas do sexo masculino, ainda segundo o autor isso pode estar relacionado com o fato das competições ainda serem enfatizadas para atletas do sexo masculino, fazendo que a mulher, ao competir, seja muito mais cobrada: o que explicaria os índices mais elevados de ansiedade diante de esportes de competição. Além disso, pode-se também levar em consideração a cultura na qual o atleta está inserido.

No presente estudo, os questionários respondidos no período pré-competitivo revelaram preocupações de ordem competitiva, como "ficar preocupado com o resultado da competição" e "ter medo de cometer erros na competição", o que é similar aos achados de Gould, Eklund e Jackson (1993), que em pesquisa com 20 lutadores olímpicos norte-americanos, indicaram a própria competição como geradora de estresse e percepção de pressão interna. Os autores revelaram expectativas pessoais de desempenho como fatores de ansiedade e também de estresse. 0 próprio fato de que o período pré-competitivo e competitivo nas fases do treinamento, investigados em ambos os estudos, são mais intensos e exigentes quanto à qualidade técnica e a preparação física (Gould et al., 1993), o que contribui para os valores mais elevados de ansiedade. Morales et al. (2013) reforçaram essa assertiva quando reportaram que os valores de ansiedade somática e de ansiedade cognitiva de atletas de judô, determinadas via CSAI-2R, eram menores em competições não oficiais em relação a competições oficiais. Estes achados também vão de encontro ao estudo de Barbacena e Grisi (2008) que ao comparar o grau de ansiedade de atletas de natação entre duas competições constataram que na competição considerada de maior nível os escores de ansiedade e autoconfiança foram maiores. Na mesma perspectiva, Sonoo et al. (2010) analisaram o nível de ansiedade de atletas de voleibol nas fases preparatória e competitiva e verificou diferenças significativas quanto ao componente cognitivo entre as duas fases, sendo ele maior na fase competitiva. Logo podemos notar que diversos fatores podem estar contribuindo para a ansiedade dos atletas estarem em baixos níveis e ainda não se alterarem. Dentre esses fatores pode-se 
destacar a motivação em relação à competição, o atleta acreditar ter chances de alcançar as metas, considerar o nível da prova elevado, nível de treinamento físico, entre outros.

Atletas mais experientes tendem a apresentar menores índices de ansiedade do que os menos experientes (Samulski, 2002). Em um estudo com atletas de judô, Detanico e Santos (2005) encontraram diferença significativa entre os níveis de ansiedade de judocas iniciantes frente à primeira competição esportiva com atletas de judô mais experientes. Recentemente, Morales et al. (2013) também reportaram que atletas de judô de nível internacional apresentaram menor ansiedade somática e menor ansiedade cognitiva, mensuradas via CSAI-2R, em relação a atletas de nível nacional espanhol. A correlação observada em nosso estudo entre tempo de prática e ansiedade na competição, apesar de moderada, indica essa associação de menor escore de ansiedade em atletas mais experientes. Matsumoto et al. (2000), em comparação entre 63 (47 homen e 16 mulheres) atletas de judô não-elite e 89 (43 homens e 44 mulheres) de elite, observaram que a auto-confiança, medida via questionário Sport-Self-Confidence Inventory (SSCI), nos dois grupos, estava relacionada aos níveis de ansiedade, mas não indicaram efeito do sexo, quando comparados os índices do SCAT. Os autores observaram correlação negativa entre o número de ataques na luta de judô e o índice do SCAT $(r=-0,36)$ para o sexo feminino. Em contrapartida, Woodman e Hardy (2003), em meta-análise com 48 estudos, relataram que a ansiedade das mulheres é distribuída abaixo e acima do limiar estabelecido pelo Competitive State Anxiety Inventory-2 (Martens, Vealey, \& Burton, 1990), enquanto os homens, largamente, demonstram ansiedade abaixo do limiar. Poucos estudos compararam os níveis de ansiedade de atletas de judô do sexo masculino e do sexo feminino em função do período da temporada.

A baixa correlação observada no presente estudo entre a ansiedade nos dois momentos analisados indica que os atletas respondem de forma bastante diversa quanto à ansiedade na competição, isto é, não necessariamente os atletas mais ansiosos no período competitivo apresentam maior ansiedade na competição. Entretanto, os resultados revelam que o tempo de experiência na modalidade reduz valores de ansiedade em situações competitivas. Estudo prévio com investigação ao longo de dois anos sobre agressividade na modalidade indica que a prática de formas (katá) e meditação auxiliam a reduzir a agressividade do praticante (Reynes \& Lorant, 2004). Além disso, Gomes e Cruz (2001) indicam, para controle da ansiedade, o treinamento respiratório, técnicas de biofeedback, auto-instrução sobre as reais expectativas do resultado e treino de relaxamento progressivo. Essas práticas podem auxiliar as mulheres atletas na redução da ansiedade durante a competição. Simultaneamente, lutadores do sexo masculino podem utilizar o treinamento mental como prática prévia ao combate competitivo, visando elaborar táticas de luta (Vorraber, 2010). A aplicação prática dessas indicações visa potencializar o desempenho dos atletas.

É importante ressaltar que os treinadores, os pais, e todos os outros profissionais envolvidos com o contexto esportivo devem respeitar a idade destes atletas, seus limites psicológicos e biológicos, pois estes podem influenciar na qualidade das experiências esportivas destes jovens, principalmente no desenvolvimento do seu autoconceito e do controle emocional, podendo afetar ou não o desenvolvimento destes atletas no esporte. Além disso, aponta-se como uma limitação do estudo não ter investigado outras classes de idade, como sênior e junior. Isso sugere que futuros trabalhos possam mensurar a ansiedade em razão da classe de idade nos dois momentos competitivos e, talvez, em diferentes níveis competitivos o que também pode influênciar o grau de importância dada pelo atleta para a competição e, por sua vez, os níveis de ansiedade.

\section{5.- Considerações finais}

Em conclusão, os resultados do presente estudo revelaram que as mulheres apresentaram maior ansiedade durante as competições do que no momento pré-competitivo. Não houve diferença entre os sexos no momento pré-competitivo. Basicamente, as atletas responderam de forma bastante ansiosa à competição, i.e., aumentaram significantemente os níveis de ansiedade neste momento em relação a si próprias no período pré-competitivo e em relação ao grupo masculino nos dois momentos. Esses dados sugerem que o trabalho preparatório durante o dia da competição deve ser diferente entre o masculino e o feminino, pois enquanto no feminino seria preciso dar 
atenção ao controle da ansiedade, no masculino outros aspectos poderiam ser analisados / considerados na preparação do atleta no dia da competição, dado que a ansiedade não parece ser afetada por este evento. Para controle da ansiedade, é recomendado o treinamento respiratório, técnicas de biofeedback, auto-instrução sobre as reais expectativas do resultado e treino de relaxamento progressivo. Em razão da menor ansiedade em competição, os homens podem utilizar o treinamento mental como prática prévia ao combate competitivo, visando elaborar a tática da luta. A aplicação prática dessas indicações visa potencializar o desempenho dos atletas. Assim, futuros estudos que considerem o nível da competição e o grau de experiência do participante devem ser conduzidos.

\section{Referências}

Barbacena, M. M., \& Grisi, R. N. F. (2008) Nível de ansiedade pré-competitiva em atletas de natação. Conexões, 6(1), 31-39.

Bertuol, L., \& Valentini, N. C. (2006). Ansiedade Competitiva de Adolescentes: gênero, maturação, nível de experiência e modalidades esportivas. Revista Educação Física/UEM, 17(1), 65-74.

Cattell, R. B., \& Scheier, I. H. (1961). The meaning and measurem ent of neuroticism and anxiety. New York: Ronald Press.

Chapman, C., Lane, A. M., Brieley, J. H., \& Terry, P. C. (1997). Anxiety, self-confidence and performance in Tae Kwon-Do. Perceptual Motor Skills, 85(2), 1275-1278.

De Rose Junior, D. (1985). Influência do grau de ansiedade-traço no aproveitamento de lances-livres. Dissertação de Mestrado. São Paulo. Universidade de São Paulo - Escola de Educação Física.

De Rose, D. (2002). A competição como fonte de estresse no esporte. Revista Brasileira de Ciência e Movimento Brasília, 10(4), 19-26.

De Rose, J. D., Sato, T. C., Selingardi, D., Bettencourt, E. L., Barros, J. C. T. S., \& Ferreira, M. C. M. (2004). Situações de jogo como fonte de "stress" em modalidades esportivas coletivas. Revista Brasileira Educação Física e Esporte, 18(4), 385-395.

Detanico, D., \& Santos, S. G. (2005). Variáveis influenciando e sendo influenciadas pela ansiedadetraço pré-competitiva: um estudo com judocas. Revista Digital Educacion Fisica y Deportes, 10(90). Consultado em http://www.efdeportes.com/efd90/judocas.htm.

Ferreira, J. S., Leite, L. P. R., \& Nascimento, C. M. C. (2010). Relação entre níveis de ansiedade-traço competitiva e idade de atletas de voleibol e análise destes níveis pré e pós-competição. Revista Motriz, 16(4), 853-857.

Figueiredo, S. H. (2000). Variáveis que interferem no desempenho do atleta de alto rendimento. Em: K. Rubio (Org.). Psicologia do esporte: interfaces, pesquisa e intervenção. São Paulo: Casa do Psicólogo.

Filaire, E., Maso, F., Sagnol, M., Ferrand, C., \& Lac, G. (2001). Anxiety, hormonal responses, and coping during a judo competition. Aggressive Behavior, 27(1), 55-63.

Franchini, E., \& Rubio, K. (2001). Aspectos Psicológicos do Judô Competitivo. Barueri (São Paulo): Manole.

Franchini, E., Matsushigue, K. A., Vecchio, F. B., \& Artioli, G. G. (2011). Physiological profiles of elite judo athletes. Sports Medicine, 41(2), 147-166.

Franchini, E., Sterkowicz, S., Meira, J. R. C. M., Gomes, F. R. F., \& Tani, G. (2008). Technical variation in a sample of high level judo players. Perceptual and Motor Skills, 106(1), 859-869.

Franchini, E., Takito, M. Y., Kiss, M. A. P. D. M., \& Sterkowicz, S. (2005). Physical fitness and anthropometrical differences between elite and non-elite judo players. Biology of Sport, $22(4), 315-328$.

Gomes, A. R. \& Cruz, J. F. A. (2001). A preparação mental e psicológica dos atletas e os factores psicológicos associados ao rendimento desportivo. Treino Desportivo, 3(16), 37-40.

Gonçalves, M. P.; Belo, R. P. (2007) Ansiedade-traço competitiva: diferenças quanto ao gênero, faixa etária, experiência em competições e modalidade esportiva em jovens atletas. Psico-USF, 12(2), 301-307.

Gould, D., Ecklund, R. C., \& Jackson, S. A. (1993). Coping strategies used by U.S. Olympic Wrestlers. Research Quartely for Exercise and Sport, 64(1), 83-93. 
IJF - International Judo Federation. (2010). Rules. Consultado em http://www.intjudo.eu/index.php?Menu=Static Page\&Action=List\&m static id=12\&lang id $=2 \&$ main $=12$.

Interdonato, G. C., Oliveira, A. R., Luiz Jr, C. C., \& Greguol, M. (2010). Analise da Ansiedade-traço Competitiva em Jovens Atletas, Conexões UNICAMP, 8(3), 1-9.

Judge, L. W., Petersen, J., \& Lydum, M. (2009). The Best Kept Secret in Sports: The 2010 Youth Olympic Games. International Review for the Sociology of Sport, 44(3), 173-191.

Julio, U. F., Takito, M. Y., Mazzei, L., Miarka, B., Sterkowicz, S., \& Franchini E. (2011). Tracking 10year competitive winning performance of judo athletes across age groups. Perceptual Motor Skills, 113(1), 139-149.

Lech, G., Sterkowicz, S., \& Rukasz, W. (2007). Significance of body height in martial arts (as exemplified by judo fighters). Human Movement, 8(1), 21-26.

Mahn, P. A. (2009). 0 efeito da ansiedade no desempenho de tenistas em uma competição. Revista Brasileira de Ciências da Saúde, 19(7), 3-8.

Martens, R. (1977). Social psychology and physical activity: problems in athletics. New York: Harper and Row.

Martens, R., Vealey, R. S., \& Burton, D. (1990). Competitive Anxiety in Sport. Champaign-Illinois: Human Kinetics.

Matsumoto, D., Takeuchi, M., Nakajima, T., \& Iida, E. (2000). Competition Anxiety, Self-Confidence, Personality and Competition Peformance of American Elite and Non-Elite Judo Athletes. Research Journal of Budo, 32(3), 12-21.

Miarka, B., Julio, U. F., Vecchio, F. B. D., Calmet, M., \& Franchini, E. (2010). Técnica y táctica en judo: Una revisión. Revista de Artes Marciales Asiáticas, 5(1), 427-431.

Miarka, B., Panissa, V., Julio, U. F., Vecchio, F. B., Calmet, M., \& Franchini, E. (2012). A comparison of time-motion performance between age groups in judo matches. Journal of Sports Sciences, 30(9), 899-905.

Moraes, L. C. (1990). Ansiedade e desempenho no esporte. Revista Brasileira de Ciência e Movimento, 4(2), 51-56.

Morales, J., Garcia, V., García-Massó, X., Salvá, P., Escobar, R., \& Buscà, B. (2013). The Use of Heart Rate Variability in Assessing Precompetitive Stress in High-Standard Judo Athletes. International Journal of Sports Medicine, 34(2), 144-51.

Reynes, E., \& Lorant, J. (2004) Competitive Martial Arts and Aggressiveness: A 2-yr. longitudinal study among young boys. Perceptual and Motor Skills, 98(1), 103-115.

Rollo, F. J. G. (2003). Habilidades Psicológicas e Ansiedade no Basquetebol de Alta Competição. Monografia em Ciências do Desporto e Educação Física, Universidade de Coimbra.

Samulski, D. M. (2002). Psicologia do Esporte. 1ạ. ed. São Paulo: Manole.

Santos, S. M. (2004). Interferência do estado emocional no desempenho competitivo de lutadores de judô. Monografia em Treinamento Desportivo, Taubaté. Universidade de Taubaté. Escola de Educação Física.

Sonoo, C. N.; Gomes, A. L.; Damasceno M. L.; Silva S. R., \& Limana, M. D. (2010) Ansiedade e desempenho: um estudo com uma equipe infantil de voleibol feminino. Motriz, Rio Claro, 16(3), 629-637.

Spielberger, C. D. (1972). Anxiety: current trends in theory and research. London: Oxford Academic Press.

Vorraber, G. A (2010). Análise dos processos cognitivo-afetivos inerentes ao desempenho táticoesportivo. Revista Brasileira de Terapia Cognitiva, 6(2), 118-141.

Woodman, T., \& Hardy, L. (2003). The relative impact of cognitive anxiety and self-confidence upon sport performance: A meta-analysis. Journal of Sports Sciences, 21(6), 443-457.

\section{Datos biográficos de los autores}

Giovanna Carla Interdonato - Faixa preta de judô, mestre em Educação Física pela Universidade Estadual de Londrina - UEL. Possui graduação em Esporte pela Universidade Estadual de Londrina. É acadêmica do curso de Fisioterapia no Centro Universitário Filadelfia - UNIFIL. Tem experiência na área de Treinamento 
Esportivo e Reabilitação para Pessoas com Deficiência. Técnica de atletas e praticantes de natação adaptada. E-mail: giointerdonato@hotmail.com.

Bianca Miarka - Faixa preta de judô, expert em análise técnico-tática em esportes de combate. Doutoranda em Educação Física, na área de Biodinâmica pela Escola de Educação Física e Esporte da Universidade de São Paulo. Possui graduação em Ciência do Esporte e especialização em Treinamento Esportivo pela Universidade Estadual de Londrina (2005) e mestrado em Educação Física pela Escola de Educação Física e Esporte da Universidade de São Paulo (2010). Tem experiência na área de Educação Física, com ênfase em Treinamento Esportivo. Possui como foco de pesquisa lutas, esportes de combate e artes marciais. E-mail: miarkasport@hotmail.com.

Emerson Franchini - Membro da comissão técnica da seleção brasileira de judô durante o período de aclimatação e durante os Jogos Olímpicos de Londres, cujos resultados representaram o melhor desempenho do Brasil nesta modalidade na história dessa competição. Professor associado (M-5) do Departamento de Esporte da Escola de Educação Física e Esporte da Universidade de São Paulo (EEFE-USP). PhD em Educação Física, na área de Biodinâmica e graduação em Educação Física (1995) pela EEFE-USP. Entrevistado pela Revista Science sobre projeto de pesquisa envolvendo análise metabólica do judô, previamente publicado no Journal of Visualized Experiments (2012). E-mail: emersonfranchini@hotmail.com. 